\title{
A MULTIPLE NONLINEAR ABEL TYPE INTEGRAL EQUATION
}

\author{
W. MYDLARCZYK
}

Communicated by Neville Ford

\begin{abstract}
We discuss a multiple nonlinear Abel type integral equation. The basic results provide criteria for the existence of nontrivial everywhere positive solutions. They are expressed in terms of the generalized Osgood condition. The global behavior of the solution, especially the conditions when it experiences blow-up, is also considered.
\end{abstract}

1. Introduction. In this paper, we examine the multiple Abel type nonlinear integral equation of the following form:

$$
\begin{array}{r}
u(t)=\int_{0}^{t}(t-s)^{\alpha_{1}-1} g_{1}(u(s)) d s+\int_{0}^{t}(t-s)^{\alpha_{2}-1} g_{2}(u(s)) d s \\
+\cdots+\int_{0}^{t}(t-s)^{\alpha_{n}-1} g_{n}(u(s)) d s \quad(t>0),
\end{array}
$$

where

$$
\begin{aligned}
& 0<\alpha_{i}, \quad i=1,2, \ldots, n, \\
& g_{i}:[0, \infty) \rightarrow[0, \infty) \text { are continuous and nondecreasing } \\
& \text { functions such that } g_{i}(0)=0 \text { and } g_{i}(z)>0 \text { for } z>0, \\
& i=1,2, \ldots, n .
\end{aligned}
$$

We are interested in the nonnegative continuous solutions. It is clear that $u \equiv 0$ is a solution to equation (1.1). It is called a trivial solution. However, only nontrivial nonnegative solutions are interesting.

2010 AMS Mathematics subject classification. Primary 45D05, Secondary 45E10, 45G05, 45G10.

Keywords and phrases. Nonlinear Abel type integral equations, the existence and uniqueness of solutions to integral Volterra equations, nontrivial solutions, the maximal solution, blow-up solutions.

Received by the editors on December 16, 2014. 
We focus on the problem of the existence of such solutions, and we investigate their properties.

Some integral equations of this type arise in one-dimensional models of the ignition and explosive behavior in diffusive media. In applications, the solution can describe a variety of processes including ones such as solid fuel combustion processes. Then the ignition in the media appears, if there exists a nontrivial continuous solution $u$ such that $u(t)>0$ for $t>0$. There are some papers related to this topic, e.g., $[5,9,11]$.

In the particular case where $n=1$, i.e., a single equation

$$
u(t)=\int_{0}^{t}(t-s)^{\alpha-1} g(u(s)) d s \quad(t>0,0<\alpha),
$$

the problems of the existence and uniqueness of the solution were exhaustively investigated, see $[\mathbf{1}, \mathbf{3}, \mathbf{4}, \mathbf{7}]$. It was shown $[\mathbf{3}, \mathbf{7}]$ that the following generalized Osgood condition

$$
\int_{0}^{\delta}\left[\frac{s}{g(s)}\right]^{1 / \alpha} \frac{d s}{s}<\infty,
$$

for any $\delta>0$ is necessary and sufficient for the existence of nontrivial solutions to equation (1.4).

The single equations of the type (1.4) each one determined by the exponent $\alpha_{i}$ and the function $g_{i}, i=1,2, \ldots, n$ are in a natural way connected with equation (1.1). Namely, using standard comparison methods for the integral Volterra type equations $[\mathbf{2}, 4]$ it can be shown that, if at least one of these equations has a nontrivial solution, then equation (1.1) has a nontrivial solution too. However, it turns out that the converse implication is not true. We discuss this problem and formulate a new integral criterion for the existence of nontrivial solutions. To present our results, we denote

$$
\phi(z)=\min \left\{\left(\frac{z}{g_{1}(z)}\right)^{1 / \alpha_{1}},\left(\frac{z}{g_{2}(z)}\right)^{1 / \alpha_{2}}, \ldots,\left(\frac{z}{g_{n}(z)}\right)^{1 / \alpha_{n}}\right\}
$$

for $z>0$, and we show that the necessary and sufficient condition for the existence of nontrivial solutions to equation (1.1) can be expressed 
in a form of the integral condition:

$$
\int_{0}^{\delta} \phi(z) \frac{d z}{z}<\infty
$$

for $\delta>0$.

Another problem of our interest concerns the explosive character of the solutions. We say that the solution blows up at some finite moment of time $0<\widehat{t}<\infty$, if $u(t) \rightarrow \infty$, as $t \rightarrow \widehat{t}-$. For equation (1.4), this problem was widely investigated $[\mathbf{5}, \mathbf{6}, \mathbf{8}, \mathbf{9}, \mathbf{1 0}]$ and the appropriate necessary and sufficient condition was stated in the form

$$
\int_{0}^{\infty}\left[\frac{s}{g(s)}\right]^{1 / \alpha} \frac{d s}{s}<\infty .
$$

In the case of equation (1.1) we provide a new integral criterion which states that the blowing-up solutions appear if and only if

$$
\int_{0}^{\infty} \phi(z) \frac{d z}{z}<\infty .
$$

In our considerations, we do not assume any Lipschitz regularity of the functions $g_{i}, i=1,2, \ldots, n$ and our arguments are based mainly on an application of monotonical and comparison methods.

2. Notation and auxiliary facts. For the reader's convenience, we collect some basic properties of the solution to the integral equations with monotone nonlinearity of the following type:

$$
u(t)=\int_{0}^{t} k(t-s, u(s)) d s,
$$

where the kernel $k$ satisfies the conditions

$(\alpha) k(t, u)$ is a nonnegative continuous function defined for $t \in$ $(0, \infty), u \in[0, \infty)$,

( $\beta) k(t, 0)=0$ and $k(t, u)>0$ for any $t>0$ and $u>0$,

$(\gamma) k\left(t, u_{1}\right) \leq k\left(t, u_{2}\right)$ for any $t \in(0, \infty)$ and $0 \leq u_{1} \leq u_{2}$,

( $\delta) \int_{0}^{\delta} k(t, u) d t<\infty$ for any $\delta>0$ and $u \geq 0$. 
Our interest in this class of equations is motivated by the observations that if we take

$$
k(t, u)=\sum_{i=0}^{n} k_{i}(t) g_{i}(u)
$$

then we can see that our original equation is the equation of such a type.

Let us denote

$$
\mathcal{T} w(t)=\int_{0}^{t} k(t-s, w(s)) d s,
$$

defined for any continuous nonnegative function $w(t), t \in[0, \infty)$. Unless it clearly stated otherwise, the functions $w(t)$ are assumed to satisfy $w(0)=0$.

The integral operator $\mathcal{T}$ has nice monotonical properties. Those basic ones are collected in the following

\section{Proposition 2.1.}

(i) if $v(t) \leq w(t)$ for $t \geq 0$, then $\mathcal{T} v(t) \leq \mathcal{T} w(t)$ for $t \geq 0$,

(ii) if $v(t)$ is a nondecreasing function, then $\mathcal{T} v(t)$ is also nondecreasing.

Proof. Part (i) follows from the monotonicity of the kernel $k(t, u)$ in the variable $u$ immediately.

To prove (ii), we see that

$$
\int_{0}^{t} k(t-s, v(s)) d s=\int_{0}^{t} k(s, v(t-s)) d s,
$$

for $t>0$. Since the integral on the right hand side is a nondecreasing function of the argument $t$, we get our assertion.

The convolution form of the operator $\mathcal{T}$ implies its invariance with respect to the shifts in the following sense: if $w_{c}(t)=w\left((t-c)_{+}\right)$, where $(t-c)_{+}=0$ for $t \in(0, c)$ and $(t-c)_{+}=t-c$ for $t \geq c$, then

$$
\mathcal{T} w_{c}(t)=(\mathcal{T} w)_{c}(t)
$$


for $t>0$. Moreover, the shift invariance of the operator $\mathcal{T}$ affects the nonuniqueness of the solution to equation (2.1). Namely, if $u(t)$ is a nontrivial solution to equation (2.1), then, for any $c>0$, the shifted function $u_{c}(t)$ is another nontrivial solution. Looking more deeply at the structure of nontrivial solutions we obtain

Proposition 2.2. Let $u(t)$ be a nontrivial solution to equation (2.1). Then there exists $c \geq 0$ such that $u(t)=0$ for $0 \leq t \leq c$ and $u(t)>0$ for $t>c$.

Proof. First, we note that, if $u(t)$ is positive at some $t_{0}$, then it is positive for $t>t_{0}$. This is easily seen if we look at the integrand in (2.1) which is then positive in a vicinity of $s=t_{0}$. Finally, it suffices to take $c=\sup \{t: u(s)=0,0 \leq s \leq t\}$ to get our assertion.

Since, in view of Proposition 2.2, to each nontrivial solution $u(t)$ there corresponds a nontrivial solution $\bar{u}(t)=u(t+c)$ positive for $t>0$, we further focus only on nontrivial solutions, which are positive for $t>0$. In fact, we are going to show that equation (2.1) has at most one such nontrivial solution.

We continue the discussion of the uniqueness solution problem for equation (2.1) beginning with a comparison its solution $u(t)$ positive for $t>0$ with nonnegative continuous solutions to the integral inequality

$$
v(t) \leq \int_{0}^{t} k(t-s, v(s)) d s
$$

for $t>0$.

Proposition 2.3. Let $u(t), t \in(0, \widehat{t})$ be a nonnegative continuous solution to equation (2.1) positive for $t>0$, and let $v(t), t \in(0, \widehat{t})$ be any solution to the integral inequality (2.5). Then

$$
v(t) \leq u(t)
$$

for $t \in(0, \widehat{t})$.

Proof. Let us take $c>0$, and let us compare the shift $v_{c}(t)$ of $v(t)$ with the solution $u(t)$. We observe that $0=v_{c}(t)<u(t)$ for $t \in(0, c)$. Furthermore, we note that, by Proposition 2.1, the following 
implication holds: if $t_{0}>0$ is such that $v_{c}(t) \leq u(t)$ for $0<t<t_{0}$, then

$$
\begin{aligned}
u\left(t_{0}\right)-v_{c}\left(t_{0}\right) & \geq \int_{0}^{t_{0}}\left(k\left(t_{0}-s, u(s)\right)-k\left(t_{0}-s, v_{c}(s)\right)\right) d s \\
& \geq \int_{0}^{c}\left(k\left(t_{0}-s, u(s)\right)-k\left(t_{0}-s, v_{c}(s)\right)\right) d s>0 .
\end{aligned}
$$

Hence, we conclude that $v_{c}(t) \leq u(t)$ for $t \in[0, \widehat{t})$. Now, letting $c \rightarrow 0$, we get our assertion.

As an immediate corollary, we obtain the following result concerning the uniqueness of the solution to equation (2.1).

Corollary 2.4. Equation (2.1) has at most one continuous solution $u(t)$ positive for $t>0$.

We show below that the nontrivial solution is nondecreasing.

Corollary 2.5. If $u(t) \geq 0$ is a continuous solution to equation (2.1) positive for $t>0$, then it is a nondecreasing function.

Proof. Let us define an auxiliary, nondecreasing function

$$
\bar{u}(t)=\sup \{u(s): 0 \leq s \leq t\}
$$

for $t>0$. Since it follows from Proposition 2.1 that

$$
u(s)=\mathcal{T} u(s) \leq \mathcal{T} \bar{u}(s) \leq \mathcal{T} \bar{u}(t)
$$

for $0 \leq s \leq t$, we get

$$
\bar{u}(t) \leq \mathcal{T} \bar{u}(t)
$$

for $t>0$. Now, by Proposition 2.3, we have $\bar{u}(t) \leq u(t)$ for $t>0$, which is only possible if $\bar{u}(t)=u(t)$ for $t>0$. Consequently, $u(t)$ is a nondecreasing function.

It follows from the discussion above that the solution $u(t)$ to equation (2.1) positive for $t>0$ is a maximal solution, which means that, for any other solution $v(t)$ to $(2.1)$, the inequality $v(t) \leq u(t)$ is satisfied on their common interval of the existence $[0, \widehat{t})$. We also note that, if 
equation (2.1) has only the trivial solution, then it is simultaneously the maximal solution.

For the maximal solution to equation (1.1) we obtain the following regularity result:

Proposition 2.6. The maximal solution to equation (1.1) is locally absolutely continuous.

Proof. We can assume that the maximal solution $u(t)$ is positive for $t>0$. Since it is a nondecreasing function, we can integrate by parts all the integrals in (1.1), which gives the following representation formula for the solution $u(t)$ :

$$
u(t)=\sum_{i=1}^{n} \frac{1}{\alpha_{i}} \int_{0}^{t}(t-s)^{\alpha_{i}} d g_{i}(u(s))
$$

for $t>0$. Since all the summands are locally absolutely continuous, we get our assertion.

In the following two propositions we give some remarks concerning the construction of the maximal solution to equation (2.1).

Proposition 2.7. Let $a>0$ be chosen in such a way that the function $v(t) \equiv 1$ satisfies the inequality

$$
\mathcal{T} v(t) \leq v(t)
$$

for $t \in(0, a)$. Then the limit function

$$
\bar{u}(t)=\lim _{n \rightarrow \infty} \mathcal{T}^{n} v(t)
$$

for $t \in(0, a)$ is the maximal solution to equation (2.1) on the interval $(0, a)$.

Proof. We first observe that the choice of appropriate $a$ is possible because the function $\mathcal{T} v(t)$ is continuous and $\mathcal{T} v(0)=0$. Since the iterative function sequence $v_{0}(t)=v(t), v_{n+1}(t)=\mathcal{T} v_{n}(t), n=$ $0,1,2, \ldots, t \in(0, a)$ is nonincreasing, there exists its pointwise limit $\bar{u}(t)=\lim _{n \rightarrow \infty} v_{n}(t)$. By the Lebesgue monotone convergence theorem 
we see that this limit function is a solution to equation (2.1). Furthermore, if $u(t)$ is any nontrivial solution, then

$$
u(t) \leq v(t) \equiv 1
$$

at least on some interval $\left(0, a_{1}\right)$ with $0<a_{1} \leq a$. Since then

$$
u(t)=\mathcal{T}^{n} u(t) \leq \mathcal{T}^{n} v(t)
$$

for $t \in\left(0, a_{1}\right)$ and $n=1,2, \ldots$, we conclude that $u(t) \leq \bar{u}(t)$ for $t \in\left(0, a_{1}\right)$. Moreover, by Proposition 2.3, we have $u(t) \leq \bar{u}(t)$ on their common interval of the existence, which ends the proof.

In Proposition 2.7, the maximal solution is obtained as the limit of the iterative function sequence $\left\{\mathcal{T}^{n} v(t)\right\}$ convergent to it from above. In a similar way, we can get it as a limit of the iterative function sequence convergent to it from below.

Proposition 2.8. If the nonnegative continuous function $w(t)$, such that $w(0)=0$ and $w(t)>0$ for $t>0$, satisfies the inequality

$$
w(t) \leq \mathcal{T} w(t)
$$

on some interval $(0, a)$, then the nondecreasing function sequence $\left\{\mathcal{T}^{n} w(t)\right\}$ is pointwise convergent to the maximal solution to equation

$$
u(t)=\lim _{n \rightarrow \infty} \mathcal{T}^{n} w(t),
$$

at least on some interval $\left(0, a_{1}\right)$ with $a_{1} \leq a$.

Proof. Let us define $v(t) \equiv 1$ for $t \geq 0$, and let us take $a_{1}>0$ such that

$$
\mathcal{T} v(t) \leq v(t) \equiv 1 \quad \text { and } \quad w(t) \leq v(t)
$$

for $t \in\left(0, a_{1}\right)$. Then we have

$$
w(t) \leq \mathcal{T} w(t) \leq \mathcal{T} v(t) \leq v(t)
$$

for $t \in\left(0, a_{1}\right)$. Hence, we conclude that the function sequence $\left\{\mathcal{T}^{n} w(t)\right\}$ is bounded and nondecreasing. Its pointwise limit $u(t)$ is a nontrivial solution to equation (2.1) positive for $t>0$. By uniqueness, it is a maximal solution to equation (2.1). 
3. Main results. Now we pass to the discussion of the existence solution problem for equation (1.1). We begin with stating the necessary and sufficient condition for the existence of nontrivial solutions.

Theorem 3.1. Equation (1.1) has a nontrivial solution if and only if condition (1.6) holds.

The necessity part. Let us consider a nontrivial solution $u(t)$ defined on some interval $(0, \widehat{t})$. By Corollary 2.5 , it is a nondecreasing function, and we can assume that it is positive for $t>0$. Let us introduce an auxiliary function

$$
L(t)=\frac{1}{u(t)} \int_{0}^{t} u(s) d s .
$$

Using the representation formula (2.10) we get

$$
L(t)=\frac{1}{u(t)} \sum_{i=1}^{n} \frac{1}{\left(\alpha_{i}+1\right) \alpha_{i}} \int_{0}^{t}(t-s)^{\alpha_{i}+1} d g_{i}(u(s)) .
$$

Since, by Proposition 2.6, the solution $u(t)$ is locally absolutely continuous, we see that $L(t)$ is locally absolutely continuous too, and we note that

$$
L^{\prime}(t)=1-\frac{u^{\prime}(t)}{u(t)} L(t) .
$$

We estimate each term of the sum in (3.1) using Jensen's inequality as follows

$$
1 / g_{i}(u(t)) \int_{0}^{t}(t-s)^{\alpha_{i}+1} d g_{i}(u(s)) \geq\left[\frac{\int_{0}^{t}(t-s)^{\alpha_{i}} d g_{i}(u(s))}{g_{i}(u(t))}\right]^{\alpha_{i}+1 / \alpha_{i}} .
$$

As a result, we get

$$
L(t) \geq \sum_{i=1}^{n} \frac{1}{\left(\alpha_{i}+1\right) \alpha_{i}}\left[\frac{u_{i}(t)}{u(t)}\right]^{\alpha_{i}+1 / \alpha_{i}}\left[\frac{u(t)}{g_{i}(u(t))}\right]^{1 / \alpha_{i}},
$$

where

$$
u_{i}(t)=\int_{0}^{t}(t-s)^{\alpha_{i}} d g_{i}(u(s)), \quad i=1,2, \ldots, n .
$$


Hence, we obtain the estimate

$$
\begin{aligned}
1- & L^{\prime}(t)=\frac{u^{\prime}(t)}{u(t)} L(t) \\
\geq & \frac{u^{\prime}(t)}{u(t)} \sum_{i=1}^{n} \frac{1}{\left(\alpha_{i}+1\right) \alpha_{i}}\left[\frac{u_{i}(t)}{u(t)}\right]^{\alpha_{i}+1 / \alpha_{i}}\left[\frac{u(t)}{g_{i}(u(t))}\right]^{1 / \alpha_{i}} \\
\geq & \frac{u^{\prime}(t)}{u(t)} \min _{1 \leq i \leq n}\left[\frac{u(t)}{g_{i}(u(t))}\right]^{1 / \alpha_{i}} \sum_{i=1}^{n} \frac{1}{\left(\alpha_{i}+1\right) \alpha_{i}}\left[\frac{u_{i}(t)}{u(t)}\right]^{\alpha_{i}+1 / \alpha_{i}} .
\end{aligned}
$$

Since

$$
u(t)=\frac{1}{\alpha_{1}} u_{1}(t)+\frac{1}{\alpha_{2}} u_{2}(t)+\cdots+\frac{1}{\alpha_{n}} u_{n}(t),
$$

we see that, for each $t \in(0, \widehat{t})$, we can find an index $1 \leq i_{0} \leq n$ such that

$$
\frac{u_{i_{0}}(t)}{u(t)} \geq \frac{1}{n} \alpha_{i_{0}} \geq \frac{1}{n} \min _{1 \leq i \leq n} \alpha_{i} .
$$

Hence, it follows that there exists a constant $c_{1}>0$ such that

$$
\sum_{i=1}^{n} \frac{1}{\left(\alpha_{i}+1\right) \alpha_{i}}\left[\frac{u_{i}(t)}{u(t)}\right]^{\alpha_{i}+1 / \alpha_{i}} \geq c_{1}
$$

for $t \in(0, \widehat{t})$. Combining (3.3) and (3.2), we obtain

$$
1-L^{\prime}(t) \geq c_{1} \frac{u^{\prime}(t)}{u(t)} \phi(u(t)),
$$

for $t \in(0, \widehat{t})$. Integrating the expressions on both sides of the inequality above we get

$$
c_{1} \int_{0}^{t} \phi(u(s)) \frac{u^{\prime}(s)}{u(s)} d s=\int_{0}^{u(t)} \phi(z) \frac{d z}{z} \leq(t-L(t)) .
$$

Hence, our assertion follows.

The sufficiency part. Our plan is to find a nondecreasing function $v(t)$ positive for $t>0$ such that

$$
v(t) \leq \mathcal{T} v(t)
$$

for $t>0$. Then, due to Proposition 2.8, we can conclude that equation (1.1) has a nontrivial solution. 
To facilitate the verification of relationship (3.5) we transform it to its equivalent form expressed in terms of the inverse function $v^{-1}(t)$. To this aim, we integrate by parts all the integrals constituting $\mathcal{T} v(t)$ obtaining the formula

$$
\mathcal{T} v(t)=\sum_{i=1}^{n} \frac{1}{\alpha_{i}} \int_{0}^{t}(t-s)^{\alpha_{i}} d g_{i}(v(s)) .
$$

Next, we substitute $z=v(s)$, and, as a result, we obtain

$$
\mathcal{T} v(t)=\sum_{i=1}^{n} \frac{1}{\alpha_{i}} \int_{0}^{v(t)}\left(t-v^{-1}(z)\right)^{\alpha_{i}} d g_{i}(z) .
$$

Hence, it follows that condition (3.5) is equivalent to the following expressed in terms of the inverse function $v^{-1}(t)$

$$
t \leq \sum_{i=1}^{n} \frac{1}{\alpha_{i}} \int_{0}^{t}\left(v^{-1}(t)-v^{-1}(z)\right)^{\alpha_{i}} d g_{i}(z)
$$

for $t>0$.

Now, for any nondecreasing continuous function $w(t)$ with $w(0)=0$ we define

$$
\mathcal{S} w(t)=\sum_{i=1}^{n} \frac{1}{\alpha_{i}} \int_{0}^{t}(w(t)-w(z))^{\alpha_{i}} d g_{i}(z),
$$

for $t>0$. We see that

$$
\begin{aligned}
\mathcal{S} w(t) \geq \sum_{i=1}^{n} \frac{1}{\alpha_{i}} \int_{0}^{t / 2}(w(t) & -w(z))^{\alpha_{i}} d g_{i}(z) \\
& \geq \sum_{i=1}^{n} \frac{1}{\alpha_{i}}(w(t)-w(t / 2))^{\alpha_{i}} g_{i}(t / 2),
\end{aligned}
$$

for $t>0$. In the sequel, we take

$$
w(t)=\int_{0}^{t / 2} \phi(z) \frac{d z}{z} .
$$

Since, for any $t>0$ and any $z \in(t / 2, t)$,

$$
\frac{z}{g_{i}(z)} \geq \frac{1}{2} \frac{t}{g_{i}(t)}, \quad i=1,2, \ldots, n
$$


we have

$$
\phi(z)>c_{2} \phi(t)
$$

where the constant $c_{2}=\min \left\{2^{-1 / \alpha_{i}}: i=1,2, \ldots, n\right\}$. Using the above estimate, we get

$$
w(t)-w(t / 2)=\int_{t / 4}^{t / 2} \phi(z) \frac{d z}{z} \geq c_{3} \phi(t / 2),
$$

for any $t>0$, where the constant $c_{3}=c_{2} \ln 2$.

Let us fix $t>0$ for a moment. Then there exists an index $1 \leq i_{0} \leq n$ such that

$$
\phi(t / 2)=\left[\frac{t / 2}{g_{i_{0}}(t / 2)}\right]^{1 / \alpha_{i_{0}}} .
$$

Hence, using (3.9), it follows that

$$
(w(t)-w(t / 2))^{\alpha_{i_{0}}} g_{i_{0}}(t / 2) \geq c_{4} t,
$$

where the constant $c_{4}=1 / 2 \min \left\{c_{3}^{\alpha_{i}}: i=1,2, \ldots, n\right\}$. Consequently, by (3.7), we have

$$
\mathcal{S} w(t) \geq c_{5} t
$$

for any $t>0$, where the constant $c_{5}=c_{4} \min \left\{1 / \alpha_{i}: i=1,2,3, \ldots, n\right\}$.

Finally, we observe that, if we take a constant $c_{6}$ such that

$$
c_{5} \min \left\{c_{6}^{\alpha_{i}}: i=1,2, \ldots, n\right\} \geq 1,
$$

then we get

$$
\mathcal{S}\left(c_{6} w\right)(t) \geq t
$$

for $t>0$. Therefore, if we define a function $v(t)$ by its inverse

$$
v^{-1}(t)=c_{6} w(t)
$$

then, due to (3.10), inequality (3.6) is satisfied for $t>0$, which means that

$$
v(t) \leq \mathcal{T} v(t)
$$

for $t>0$, and our assertion follows.

To illustrate the results obtained above we discuss the following integral equation. 
Example 3.2. We construct two functions $g_{1}(z)$ and $g_{2}(z)$ satisfying condition (1.3) for which the integral equation

$$
u(t)=\int_{0}^{t}(t-s)^{-2 / 3} g_{1}(u(s)) d s+\int_{0}^{t}(t-s)^{-1 / 2} g_{2}(u(s)) d s
$$

has a nontrivial solution, but neither of the two single equations

$$
u(t)=\int_{0}^{t}(t-s)^{-2 / 3} g_{1}(u(s)) d s
$$

and

$$
u(t)=\int_{0}^{t}(t-s)^{-1 / 2} g_{2}(u(s)) d s
$$

has any nontrivial solutions.

We first define a decreasing sequence $a_{0}=1, a_{i+1}=\left[\sqrt{a_{i}+1}-1\right]^{2}$ for $i=0,1,2, \ldots$ convergent to zero, as $i \rightarrow \infty$. It is easily seen that

$$
\frac{a_{i}-a_{i+1}}{\sqrt{a_{i+1}}}=2, \quad i=1,2, \ldots .
$$

Let us consider a function

$$
h(z)=1 / \sqrt{z}
$$

for $z>0$ and its piecewise linear interpolant at the knots $a_{i}$

$$
f(z)=h\left(a_{i}\right) \frac{z-a_{i+1}}{a_{i}-a_{i+1}}+h\left(a_{i+1}\right) \frac{a_{i}-z}{a_{i}-a_{i+1}},
$$

for $z \in\left[a_{i+1}, a_{i}\right), i=0,1,2, \ldots$ Since $h(z)$ is convex and $f\left(a_{i}\right)=h\left(a_{i}\right)$, $i=1,2, \ldots$, we easily check that the function $f(z)$ is nonincreasing and

$$
h(z) \leq f(z)
$$

for $z \in(0,1)$. Finally, we define:

$$
g_{1}(z)= \begin{cases}z^{2 / 3} h(z)^{-1 / 3}, & \text { if } z \in\left(a_{2 i+1}, a_{2 i}\right) \\ z^{2 / 3} f(z)^{-1 / 3}, & \text { if } z \in\left(a_{2 i+2}, a_{2 i+1}\right)\end{cases}
$$

and

$$
g_{2}(z)=\left\{\begin{array}{l}
z^{1 / 2} f(z)^{-1 / 2}, \text { if } z \in\left(a_{2 i+1}, a_{2 i}\right) \\
z^{1 / 2} h(z)^{-1 / 2}, \text { if } z \in\left(a_{2 i+2}, a_{2 i+1}\right)
\end{array}\right.
$$


$i=0,1,2,3, \ldots$. We observe that the function $\phi(z)$ for equation (3.12) has a form

$$
\phi(z)=\min \left\{\left(\frac{z}{g_{1}(z)}\right)^{3},\left(\frac{z}{g_{2}(z)}\right)^{2}\right\}=z h(z)=\sqrt{z},
$$

for $z \in(0,1)$. Thus,

$$
\int_{0}^{\delta} \phi(z) \frac{d z}{z}<\infty
$$

and consequently, equation (3.12) has a nontrivial solution by Theorem 3.1.

However, it follows from (3.14) that

$$
\begin{aligned}
\int_{a_{i+1}}^{a_{i}} f(z) d z & =\frac{h\left(a_{i}\right)+h\left(a_{i+1}\right)}{2}\left(a_{i}-a_{i+1}\right) \\
& \geq \frac{a_{i}-a_{i+1}}{2 \sqrt{a_{i+1}}}=1
\end{aligned}
$$

$i=1,2, \ldots$

Since

$$
\left(\frac{z}{g_{1}(z)}\right)^{3} \frac{1}{z}=f(z) \quad \text { for } z \in\left(a_{2 i+2}, a_{2 i+1}\right)
$$

and

$$
\left(\frac{z}{g_{2}(z)}\right)^{2} \frac{1}{z}=f(z) \quad \text { for } z \in\left(a_{2 i+1}, a_{2 i}\right),
$$

$i=0,1,2,3, \ldots$, it follows from (3.15) that

$$
\int_{0}^{\delta}\left(\frac{z}{g_{1}(z)}\right)^{3} \frac{1}{z} d z=\infty
$$

and

$$
\int_{0}^{\delta}\left(\frac{z}{g_{2}(z)}\right)^{2} \frac{d z}{z}=\infty
$$

for $\delta>0$ and, consequently, each integral equation (3.13a) and (3.13b) has only trivial solutions by (1.5). 
Our proof of the above theorem is based on some a priori estimates of the maximal solution to equation (1.1), which can be stated explicitly as follows:

Corollary 3.3. Let $u(t)$ be a maximal solution to equation (1.1) positive for $t>0$. Then there exist constants $c_{m}$ and $c_{M}>0$ such that

$$
c_{m} \int_{0}^{t} \phi(z) \frac{d z}{z} \leq u^{-1}(t) \leq c_{M} \int_{0}^{t / 2} \phi(z) \frac{d z}{z} .
$$

for $t>0$.

Proof. The left-hand inequality follows from (3.4) immediately.

To prove the right-hand inequality we come back to the function $v(t)$ constructed in the proof of the sufficiency part of Theorem 3.1. Since it satisfies the inequality $v(t) \leq \mathcal{T} v(t)$ for $t>0$, by Proposition 2.3, we get

$$
v(t) \leq u(t)
$$

for $t>0$. Passing to the inverse functions, this inequality can be rewritten in the form

$$
u^{-1}(z) \leq v^{-1}(z)
$$

for $z>0$. Thus, our assertion follows from the definition of $v^{-1}(t)$ given in (3.8) and (3.11).

As an immediate consequence of the a priori estimates above we obtain the following results concerning the blowing up behavior of the maximal nontrivial solution.

Corollary 3.4. The maximal nontrivial solution $\bar{u}(t)$ to equation (1.1) blows up if and only if the condition (1.8) holds.

Proof. Let us consider the inverse function $\bar{u}^{-1}(t)$. We note that $\bar{u}(t)$ has a blowing up behavior if and only if there exists a finite limit

$$
\lim _{t \rightarrow \infty} \bar{u}^{-1}(t)<\infty .
$$

Hence, our assertion follows from the a priori estimates given in Corollary 3.3 . 
The result obtained concerning the blow up behavior of the maximal solution is discussed in the following example.

Example 3.5. We construct two functions $g_{1}(z)$ and $g_{2}(z)$ satisfying (1.3) for which the integral equation

$$
u(t)=\int_{0}^{t}(t-s)^{-1 / 3} g_{1}(u(s)) d s+\int_{0}^{t}(t-s)^{-1 / 2} g_{2}(u(s)) d s
$$

has a blowing up nontrivial solution, while each of the two single equations

$$
u(t)=\int_{0}^{t}(t-s)^{-1 / 3} g_{1}(u(s)) d s
$$

and

$$
u(t)=\int_{0}^{t}(t-s)^{-1 / 2} g_{2}(u(s)) d s
$$

has nontrivial solutions, but none of them blows up.

We first define an increasing sequence $b_{0}=1, b_{i+1}=2 b_{i}^{2}+b_{i}$, which is divergent, as $i \rightarrow \infty$. It is easily seen that

$$
\frac{b_{i+1}-b_{i}}{b_{i}^{2}}=2, \quad i=1,2, \ldots .
$$

Let us consider a function

$$
h(z)=1 / z^{2}
$$

for $z>1$ and its piecewise linear interpolant at the knots $b_{i}$

$$
f(z)=h\left(b_{i+1}\right) \frac{z-b_{i}}{b_{i+1}-b_{i}}+h\left(b_{i}\right) \frac{b_{i+1}-z}{b_{i+1}-b_{i}}
$$

for $z \in\left[b_{i}, b_{i+1}\right), i=0,1,2, \ldots$. Since $h(z)$ is convex and $f\left(b_{i}\right)=h\left(b_{i}\right)$, $i=1,2, \ldots$, we easily check that the function $f(z)$ is nonincreasing and

$$
h(z) \leq f(z)
$$

for $z>1$. Finally, we define the following two functions:

$$
g_{1}(z)=\left\{\begin{array}{l}
z^{1 / 3} h^{-2 / 3}(z), \text { if } z \in\left(b_{2 i}, b_{2 i+1}\right), \\
z^{1 / 3} f^{-2 / 3}(z), \text { if } z \in\left(b_{2 i+1}, b_{2 i+2}\right)
\end{array}\right.
$$


and

$$
g_{2}(z)=\left\{\begin{array}{l}
z^{1 / 2} f^{-1 / 2}(z), \text { if } z \in\left(b_{2 i}, b_{2 i+1}\right), \\
z^{1 / 2} h^{-1 / 2}(z), \text { if } z \in\left(b_{2 i+1}, b_{2 i+2}\right),
\end{array}\right.
$$

$i=0,1,2,3, \ldots$. We observe that the function $\phi(z)$ for equation (3.16) has a form

$$
\phi(z)=\min \left\{\left(\frac{z}{g_{1}(z)}\right)^{3 / 2},\left(\frac{z}{g_{2}(z)}\right)^{2}\right\}=z h(z)=1 / z,
$$

for $z>1$.

To complete the construction, we define the parts of the functions $g_{1}$ and $g_{2}$ on the interval $[0,1]$ similarly as in Example 3.2. Thus,

$$
\int_{0}^{\infty} \phi(z) \frac{d z}{z}<\infty
$$

and consequently, equation (3.16) has a blowing up solution.

However, it follows from (3.18) that

$$
\int_{b_{i}}^{b_{i+1}} f(z) d z=\frac{h\left(b_{i}\right)+h\left(b_{i+1}\right)}{2}\left(b_{i+1}-b_{i}\right) \geq \frac{b_{i+1}-b_{i}}{2 b_{i}^{2}}=1
$$

for $i=1,2, \ldots$.

Since

$$
\left(\frac{z}{g_{1}(z)}\right)^{3 / 2} \frac{1}{z}=f(z) \quad \text { if } z \in\left(b_{2 i+1}, b_{2 i+2}\right)
$$

and

$$
\left(\frac{z}{g_{2}(z)}\right)^{2} \frac{1}{z}=f(z) \quad \text { if } z \in\left(b_{2 i}, b_{2 i+1}\right),
$$

for $i=0,1,2,3, \ldots$, it follows from (3.19) that

$$
\int_{0}^{\infty}\left(\frac{z}{g_{1}(z)}\right)^{3 / 2} \frac{d z}{z}=\infty
$$

and

$$
\int_{0}^{\infty}\left(\frac{z}{g_{2}(z)}\right)^{2} \frac{d z}{z}=\infty
$$


for $\delta>0$ and, consequently, by (1.7), the nontrivial solutions of each integral equation (3.17a) and (3.17b) do not blow up.

\section{REFERENCES}

1. P.J. Bushell and W. Okrasiński, Uniqueness of solutions for a class of nonlinear Volterra integral equations with convolution kernel, Math. Proc. Camb. Phil. Soc. 106 (1989), 547-552.

2. C. Corduneanu, Integral equations and applications, Cambridge University Press, Cambridge, 1991.

3. G. Gripenberg, Unique solutions of some Volterra integral equations, Math. Scand. 48 (1981), 59-67.

4. G. Gripenberg, S.O. Londen and O. Staffans, Volterra integral and functional equations, Encycl. Math. Appl. 34, Cambridge University Press, New York, 1990.

5. D.G. Lasseigne and W.E. Olmstead, Ignition or nonignition of a combustible solid with marginal heating, Quart. Appl. Math. 49 (1991), 303-312.

6. T. Małolepszy and W. Okrasiński, Conditions for blow-up of solutions of some nonlinear Volterra integral equations, J. Comp. Appl. Math. 205 (2007), 744-750.

7. W. Mydlarczyk, The existence of nontrivial solutions of Volterra equations, Math. Scand. 68 (1991), 83-88.

8. , A condition for finite blow-up time for a Volterra equations, J. Math. Anal. Appl. 181 (1994), 248-253.

9. W.E. Olmstead, Ignition of a combustible half space, SIAM J. Appl. Math. 43 (1983), 1-15.

10. C.A. Roberts, Analysis of explosion for nonlinear Volterra equations with blow-up solutions, J. Comp. Appl. Math. 97 (1998), 153-166.

11. C.A. Roberts, D.G. Lassigne and W.E. Olmstead, Volterra equations which models explosion in a diffusive medium, J. Int. Equat. Appl. 5 (1993), 531-546.

Faculty of Pure and Applied Mathematics, Technical University of Wroceaw, Wybrzeze Wyspianskiego 27, 50-370 WrocŁaW, Poland

Email address: wojciech.mydlarczyk@pwr.edu.pl 